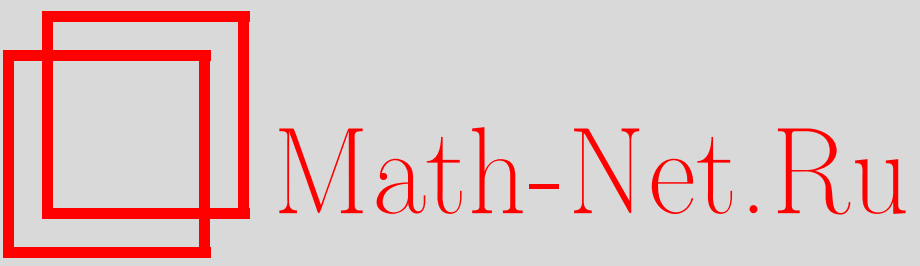

С. В. Червон, Ю. А. Свистунова, Сферически-симметричная нелинейная сигмамодель: точные решения, найденные с использованием метода изометрических погружений, Вестн. Сам. гос. техн. ун-та. Сер. Физ.-мат. науки, 2011, выпуск 2()$, 227-234$

DOI: https://doi.org/10.14498/vsgtu874

Использование Общероссийского математического портала Math-Net.Ru подразумевает, что вы прочитали и согласны с пользовательским соглашением

http://www.mathnet.ru/rus/agreement

Параметры загрузки:

IP : 35.174 .16 .151

26 апреля 2023 г., 14:01:17 
УДК 530.12

\title{
СФЕРИЧЕСКИ-СИММЕТРИЧНАЯ НЕЛИНЕЙНАЯ СИГМА-МОДЕЛЬ: ТОЧНЫЕ РЕШЕНИЯ, НАЙДЕННЫЕ С ИСПОЛЬЗОВАНИЕМ МЕТОДА ИЗОМЕТРИЧЕСКИХ ПОГРУЖЕНИЙ
}

\section{С. В. Червон, Ю.А. Свистунова}

Ульяновский государственный педагогический университет им. И. Н. Ульянова, 432000, Ульяновск, пл. 100-летия со дня рождения В. И. Ленина, 4.

E-mails: chervon.sergey@gmail.com, u.a.svistunova@gmail.com

\begin{abstract}
Предложен метод построения точных космологических решений в рамках сферически-симметричной нелинейной сигма-модели, основанный на изометрических погружениях пространства целей (киралъного пространства) в пространство-время. Рассматривается применение метода изометрических погружений в рамках двух- и трёхкомпонентных киральных пространств, погружаемых в четырёхмерное пространство-время погружения. Найдены точные космологические решения для специальных случаев двух- и трёхкомпонентных нелинейных сигма-моделей со сферической симметрией, проводится анализ полученных решений.
\end{abstract}

Ключевые слова: нелинейная сигма-модель, метод изометрического погружения, точные космологические решения.

Введение. Задача получения точных решений в рамках нелинейной сигма-модели (НСM) со сферической симметрией впервые была поставлена в работах Г. Г. Иванова $[1,2]$. Однако в рамках данных работ были рассмотрены только двумерные $\mathrm{SO}(3) \mathrm{HCM}$, для которых и были найдены некоторые классы точных решений. Для трёхмерных киральных пространств самогравитирующей НСМ ввиду сложности получаемой самосогласованной системы уравнений киральных полей и уравнений Эйнштейна точные решения со сферической симметрией до настоящего времени не были получены.

В настоящей работе представлены точные космологические решения в рамках трёх- и двухкомпонентных самогравитирующих сферически-симметричных НСМ, найденные с помощью метода изометрического погружения (ИП).

В общем случае методы поиска точных решений НСМ как источника гравитационного поля можно условно разделить на три группы. Первую составляют так называемые прямые методы, т. е. непосредственное решение самосогласованной системы полевых уравнений $\mathrm{HCM}$ и уравнений Эйнштейна для заданной метрики пространства-времени и кирального пространства. Второй подход [1] основывается на установлении связей между изометрическими движениями в пространстве-времени и в пространстве целей. При этом векторы Киллига данных пространств соотносятся друг с другом через анзац Иванова [3]. Третья группа включает в себя методы, базирующиеся на погружении изометрическим образом пространства целей в пространство-время. За счёт выбора связей между киральными полями и пространственновременными координатами самосогласованная система полевых уравнений

Сергей Викторович Червон (д.ф.-м.н., проф.), профессор, каф. физики. Юлия Александровна Свистунова, научный сотрудник, каф. физики. 
НСМ и уравнений Эйнштейна упрощается, что позволяет найти точные решения такой системы. Как известно, чисто кинетическая нелинейная сигмамодель в математической литературе выступает под названием гармонического отображения. Связь между гармоническими отображениями и изометрическими погружениями исследована в работах $[4,5]$. Отметим также, что в работе [6] рассмотрены общие подходы к исследованию самогравитирующих HCM с учётом изометрических погружений. В данной работе используется метод ИП для получения точных решений самогравитирующей сферическисимметричной НСМ.

Нелинейная сигма-модель как источник гравитации и теорема Кэмпбелла-Магаарда. Впервые самогравитирующие НСМ были введены Г. Г. Ивановым [1] (см. также [7]). Самогравитирующая чисто кинетическая НСМ [8] определяется действием вида

$$
S=\int \sqrt{-g} d^{4} x\left(\frac{R}{2 \kappa}+\frac{1}{2} h_{A B}(\varphi) \varphi_{, \mu}^{A} \varphi_{, \nu}^{B} g^{\mu \nu}\right) .
$$

Здесь $\left(\mathcal{M}^{4}, g_{\mu \nu}(x)\right)$ - пространство-время; $\left(\mathcal{N}^{n}, h_{A B}(\varphi)\right)$ - пространство целей (киральное пространство), криволинейные координаты которого $\varphi=\left(\varphi^{1}, \varphi^{2}\right.$, $\left.\ldots, \varphi^{n}\right)$ соответствуют компонентам кирального поля; $R$ - скалярная кривизна; $\kappa$ - гравитационная постоянная Эйнштейна. Частные производные обозначены так: $\partial_{k} \varphi^{A}=: \varphi_{, k}^{A}$. Запишем уравнения Эйнштейна в следующем виде:

$$
R_{\mu \nu}=\kappa\left(T_{\mu \nu}-\frac{1}{2} g_{\mu \nu} T\right)=\kappa h_{A B} \varphi_{, \mu}^{A} \varphi_{, \nu}^{B},
$$

где $\kappa$-гравитационная постоянная Эйнштейна. Здесь использовались компоненты тензора энергии-импульса НCM (1):

$$
T_{\mu \nu}=h_{A B} \varphi_{, \mu}^{A} \varphi_{, \nu}^{B}-g_{\mu \nu}\left(\frac{1}{2} h_{A B} \varphi_{, \gamma}^{A} \varphi_{,}^{B} g^{\gamma \delta}\right) .
$$

Варьирование действия (1) по полям $\varphi^{A}$ приводит к полевым уравнениям

$$
\frac{1}{\sqrt{-g}} \partial_{\mu}\left(\sqrt{-g} g^{\mu \nu} h_{A B} \varphi_{,}^{B}\right)-\frac{1}{2} \frac{\partial h_{B C}}{\partial \varphi^{A}} \varphi_{,{ }_{\mu}}^{B} \varphi_{,}^{C}{ }_{\nu}^{\mu \nu}=0 .
$$

Таким образом динамика самогравитирующей НСМ определяется системой уравнений (2), (3).

1. Метод изометрического погружения. Основой метода изометрического погружения является теорема Кэмпбелла-Магаарда о погружении.

Теорема. Любое $n$-мерное (псевдо-)риманово многообразие $\left(\mathcal{N}^{n}, g\right)$ может быть локально и изометрически погружено в $(n+1)$-мерное Риччиплоское многообразие $\left(\mathcal{M}^{n+1}, \bar{g}\right)[12],[13]$.

Теорема Кэмпбелла-Магаарда сформулирована для римановых многообразий, т. е. для положительно определённых метрик. Однако в работе Дайя и Ромеро $[9,10]$ показано, что теорему можно распространить на псевдо-римановы многообразия. По аналогии с работами $[9,10]$ в [14] сделано обобщение теоремы о погружении на случай пространств Эйнштейна с ненулевым тензором Риччи. Отметим, что в работе [11] представлено обобщение теоремы 
Кэмпбелла-Магаарда на случай Риччи-плоских пространств-времён со скалярным полем.

2. Трёхкомпонентные сферически-симметричные модели. В работе [6] сделано обобщение теоремы Кэмпбелла-Магаарда о погружении [12], [13] на случай мультиплета киральных полей. На основе данного обобщения и по аналогии с работой [6] рассмотрим приложение метода ИП для трёхкомпонентной НСМ со сферической симметрией.

В общем случае алгоритм применения метода ИП состоит в следующем. Рассматриваем самогравитирующую НСМ в пространстве-времени с метрикой представленной в нормальных гауссовых координатах:

$$
d S_{M}^{2}=\bar{g}_{\alpha \beta}\left(x^{\alpha}, x^{3}\right) d x^{\alpha} d x^{\beta}+\varepsilon\left(x^{\alpha}, x^{3}\right)\left(d x^{3}\right)^{2},
$$

где $\alpha, \beta$ пробегают значения $0,1,2, \varepsilon= \pm 1$. Здесь и далее будем использовать обозначения из работы [6].

Киральное пространство характеризуется метрикой, которая строится как трёхмерная часть базового пространства-времени погружения (4):

$$
d S_{N}^{2}=h_{\alpha \beta}\left(\varphi^{\gamma}\right) d \varphi^{\alpha} d \varphi^{\beta} .
$$

При этом определяется соответствия между киральными полями и координатами погружаемого пространства, а также компонентами метрик:

$$
\varphi^{\gamma}=x^{\gamma} ; \quad h_{\alpha \beta}=g_{\alpha \beta} ; \quad g_{\alpha \beta}\left(x^{\gamma}\right)=\bar{g}_{\alpha \beta}\left(x^{\gamma}, 0\right) .
$$

Тогда в соответствии с методом ИП пространство-время погружения характеризуется метрикой

$$
d S_{M}^{2}=g_{\alpha \beta}\left(x^{\alpha}, x^{3}\right)\left(\omega_{0}^{2}\left(x^{3}\right)^{2} \pm 2 \omega_{0} x^{3}+1\right) d x^{\alpha} d x^{\beta}+\varepsilon\left(d x^{3}\right)^{2},
$$

где $\omega_{0}=$ const - некоторая постоянная.

Применим стандартную технику метода ИП для сферически-симметричных НCM, которые, как будет продемонстрировано ниже, могут быть изометрически погружены в пространства-времена, также обладающие сферической симметрией.

Итак, выбираем метрику сферически-симметричного пространства-времени в полярных гауссовых координатах:

$$
d s^{2}=h(r, t) d t^{2}-d r^{2}-r^{2} f(r, t)\left(d \theta^{2}+\sin ^{2} \theta d \phi^{2}\right),
$$

где $h(r, t)$ и $f(r, t)$ - некоторые функции времени и координат.

На основе пространства-времени (8) можно построить два класса трёхкомпонентных сферически-симметричных НСМ. Первый класс моделей отвечает выбору $\bar{g}_{33}=-1$ и $x^{3}=r$. Второй класс включает в себя частный случай для пространства-времени $(8)$, когда $h(r, t)=\mathrm{const}=1$. В этом случае метрика имеет вид

$$
d s^{2}=d t^{2}-d r^{2}-r^{2} f(r, t)\left(d \theta^{2}+\sin ^{2} \theta d \phi^{2}\right) .
$$

Тогда можно выбрать $\bar{g}_{33}=1$ и $x^{3}=t$. 
Рассмотрим общее решение, когда на функции $h(r, t)$ и $f(r, t)$ не накладываются дополнительные условия. Пространство целей трёхкомпонентной модели, построенное как трёхмерная часть сферически-симметричного пространства-времени, может быть записано в следующем виде:

$$
d S_{c h}^{2}=h\left(r, \varphi^{0}\right) d\left(\varphi^{0}\right)^{2}-r^{2} f\left(r, \varphi^{0}\right)\left(d\left(\varphi^{1}\right)^{2}-\sin ^{2}\left(\varphi^{1}\right) d\left(\varphi^{2}\right)^{2}\right) .
$$

Однако точные решения в рамках данной модели удаётся найти только для случая $h(r, t)=$ const и $f(r, t)=R(r) T(t)$. Сразу полагаем $h=1$, поскольку всегда можно выполнить преобразование $t \rightarrow t \sqrt{h}$.

Возможны два случая.

a) Рассмотрим $\bar{g}_{33}=-1$ и $x^{3}=r$. В этом случае для функций $h(r, t)$ и $f(r, t)$, входящих в состав метрики, решение можно записать в виде

$$
\begin{gathered}
h(r, t)=h=1 \\
f(r, t)=R(r) T(t)=\frac{\lambda}{r^{2}}\left[\frac{1}{\sqrt{\kappa}}\left(C_{2} \cos (t \sqrt{\kappa})-C_{1} \sin (t \sqrt{\kappa})\right)-\frac{2}{\lambda \kappa}\right],
\end{gathered}
$$

где $\omega_{0}^{2}=\kappa / 2 ; C_{1}, C_{2}, \lambda$ - константы, причём $C_{1}^{2}+C_{2}^{2}=4 /\left(\lambda^{2} \kappa\right) \neq 0$, $\lambda \neq 0$, или, если $C_{1}=0$, то $C_{2} \neq 0$, также при $C_{2}=0$ имеем $C_{1} \neq 0$.

б) При $h=$ const $=1$ можно выбрать $\bar{g}_{33}=1$ и $x^{3}=t$. Тогда метрика кирального пространства, построенного на трёхмерной части пространства-времени, будет отличаться от (10) и иметь вид

$$
d S_{c h}^{2}=-d\left(\varphi^{0}\right)^{2}-\left(\varphi^{0}\right)^{2} f\left(r, \varphi^{0}\right)\left(d\left(\varphi^{1}\right)^{2}-\sin ^{2} \varphi^{1} d\left(\varphi^{2}\right)^{2}\right) .
$$

Пространство-время, в которое изометрически погружается (11), как показано в [6], представляется таким образом:

$$
d s_{t}^{2}=-\left(\omega_{0} t-1\right)^{2}\left(d r^{2}+r^{2} f(r, t)\left(d \theta^{2}+\sin ^{2} \theta d \phi^{2}\right)\right)+d t^{2} .
$$

Решая систему полевых уравнений и уравнений Эйнштейна с учётом соотношения координат кирального пространства и пространства погружения, определяемое методом ИП, получаем ограничения на функцию $f(r, t)=f(r)$ и два класса решений:

$$
\begin{aligned}
& \text { 1) } f(r, t)=f(r)=\frac{2}{\zeta r^{2}}\left(\cos (2 r \sqrt{2 \zeta})-\cos ^{2}\left(r \frac{\zeta}{2}\right)\right) \text {; } \\
& \text { 2) } f=f(r, t)=f(r)=\frac{2}{\zeta r^{2}} \cos ^{2}\left(r \frac{\zeta}{2}\right),
\end{aligned}
$$

где $\zeta=2 \kappa-4 \omega_{0}^{2}$. Таким образом, имеем два семейства киральных пространств, соответствующих каждому значению функции $f(r)$. Если ввести функции

$$
R^{2}(r, t)=\left(\omega_{0}^{2} t-1\right)^{2}(\cos (r \sqrt{2 \zeta}) \pm 1), \quad \rho^{2}(r)=\frac{2(\cos (r \sqrt{2 \zeta}) \pm 1}{\zeta \sin (r \sqrt{2 \zeta})}
$$


то пространства погружения будут представлять собой не что иное, как неоднородные, изотропные, сферически-симметричные пространства-времена Толмена [15].

3. Двухкомпонентная сферически-симметричная нелинейная сигма-модель как источник гравитации. Выберем метрику пространства-времени в виде

$$
d S_{M}^{2}=\bar{g}_{a b}\left(x^{c}, x^{J}\right) d x^{a} d x^{b}+\bar{g}_{I K}\left(x^{c}, x^{J}\right) d x^{I} d x^{K},
$$

где индексы $a, b, c$ пробегают значения 0,1, а $I, J, K$ - значения 2,3 . Модель можно построить на любых двух компонентах метрики $\left(\right.$ на $\bar{g}_{a b}\left(x^{c}, x^{J}\right) d x^{a} d x^{b}$ или на $\left.\bar{g}_{I K}\left(x^{c}, x^{J}\right) d x^{I} d x^{K}\right)$.

Применим метод ИП для исследования $\bar{g}_{I K}\left(x^{c}, x^{J}\right) d x^{I} d x^{K}$-части. При исследовании $\bar{g}_{a b}\left(x^{c}, x^{J}\right) d x^{a} d x^{b}$-части результат будет таким же, с учётом поправки на индексы.

Рассмотрим метрику пространства целей

$$
d S_{N}^{2}=h_{a b}\left(\varphi^{c}\right) d \varphi^{a} d \varphi^{b} .
$$

Определим метрику (12) для применения метода ИП как

$$
d S_{M}^{2}=\bar{g}_{a b}\left(x^{c}, x^{2}\right) d x^{a} d x^{b}+\epsilon\left(d x^{2}\right)^{2}+F\left(x^{c}, x^{J}\right)\left(d x^{3}\right)^{2} .
$$

Таким образом, получаем следующие соотношения:

$$
\varphi^{c}=x^{c} ; \quad h_{a b}=g_{a b}, \quad g_{a b}\left(x^{c}\right)=\bar{g}_{a b}\left(x^{c}, 0\right) .
$$

Компоненты ТЭИ имеют вид

$$
T_{i k}=g_{a b} \delta_{i}^{a} \delta_{k}^{b}-\frac{1}{2} \bar{g}_{i k}\left(g_{c d} \bar{g}^{c d}\right) .
$$

В случае метрики (14) компоненты ТЭИ упрощаются:

$$
T_{a b}=g_{a b}-\frac{1}{2} \bar{g}_{a b}\left(g_{c d} \bar{g}^{c d}\right), \quad T_{22}=-\frac{1}{2} \epsilon\left(g_{c d} \bar{g}^{c d}\right), \quad T_{33}=-\frac{1}{2} F\left(x^{c}, x^{J}\right)\left(g_{c d} \bar{g}^{c d}\right) .
$$

Уравнения киральных полей запишутся так:

$$
\frac{1}{\sqrt{|\bar{g}|}} \partial_{c}\left(\sqrt{|\bar{g}|} \bar{g}^{c d} g_{a d}\right)-\frac{1}{2} \frac{\partial g_{b c}}{\partial \varphi^{a}} \bar{g}^{b c}=0
$$

Связь между двухмерной частью пространства-времени $\bar{g}_{a b}\left(x^{c}, x^{J}\right) d x^{a} d x^{b}$ и пространством целей определим как

$$
\bar{g}_{a b}\left(x^{c}, x^{2}\right)=\Omega^{2}\left(x^{2}\right) g_{a b}, \quad \Omega^{2}(0)=1 .
$$

Решая полевые уравнения (15), находим

$$
T_{a b}=0 ; \quad T_{22}=-\epsilon \frac{1}{\Omega^{2}} ; \quad T_{33}=-\frac{F}{\Omega^{2}} ; \quad T=-\frac{2}{\Omega^{2}} .
$$


Уравнения Эйнштейна могут быть представлены в виде

$$
\bar{R}_{a b}=\frac{g_{a b}}{\Omega^{2}} ; \quad \bar{R}_{22}=\frac{\epsilon}{\Omega^{2}} ; \quad \bar{R}_{33}=\frac{F}{\Omega^{2}} .
$$

Из решений полевых уравнения (15), а также из условия, что все двумерные пространства являются эйнштейновскими, можно показать, что решения существуют только в том случае, когда

$$
\Omega=\Omega_{0}=\text { const }=1 \text {. }
$$

Решения для функции $F$ принимают вид:

1) при $\epsilon=1: F=F_{0}\left(x^{3}\right)\left[\cos ^{2}\left(\frac{x_{0}^{2}-x^{2}}{\Omega_{0}}\right)\right]$;

2) при $\epsilon=-1: F=F_{0}\left(x^{3}\right)\left[\cosh ^{2}\left(\frac{x_{0}^{2}-x^{2}}{\Omega_{0}}\right)\right]$,

где $F_{0}\left(x^{3}\right), x_{0}^{2}$ - некоторые начальные граничные значения функции и координаты.

Таким образом, получены точные решения в рамках двумерной НСМ.

4. Сферически-симметричная двухкомпонентная нелинейная сигма-модель. Рассмотрим примеры получения точных решений с использованием метода ИП. Для этого строим двухкомпонентную $\mathrm{HCM} h_{a b}\left(\varphi^{c}\right)$ как двумерную часть метрики сферически-симметричного исходного пространства-времени $g_{a b}\left(x^{c}, x^{2}, x^{3}\right)$, которое в нормальных гауссовых координатах может быть представлено как

$$
d s^{2}=h(r, t) d t^{2}-d r^{2}-r^{2} f(r, t)\left(d \theta^{2}+\sin ^{2} \theta d \phi^{2}\right),
$$

где $h(r, t)$ и $f(r, t)$ - некоторые функции времени и координат. Другими словами:

$$
h_{a b}\left(\varphi^{c}\right)=g_{a b}\left(x^{c}\right) ; \quad \varphi^{c}=x^{c} ; \quad g_{a b}\left(x^{c}\right)=\bar{g}_{a b}\left(x^{c}, x^{2}=0, x^{3}=0\right) .
$$

В рамках данной модели рассмотрим два семейства решений:

1) в общем случае выбираем: $x^{2}=r, \epsilon=-1$;

2) частный случай, когда $h(r, t)=h=$ const. Тогда можно выбрать в качестве $x^{2}$ время, т. е. $x^{2}=t, \epsilon=1$.

Параметры метрик погружаемых пространств и пространств погружения, для которых были получены точные решения, приведены в таблице.

Параметры решений для двухкомпонентной сферически-симметричной НСМ

\begin{tabular}{c|c|c|c|c}
\hline Параметры $f(r, t), h(r, t)$ & $\epsilon$ & $x^{3}$ & $x^{2}$ & $F\left(x^{C}, x^{J}\right)$ \\
\hline$f(r, t) ; h(r, t)=F\left(x^{C}, x^{J}\right)$ & -1 & $t$ & $r$ & $h_{0}(t) \operatorname{ch}^{2}(-r)$ \\
$f(r, t)=\left(F_{0} / r^{2}\right) \cos ^{2} t, F_{0}=\mathrm{const} ; h(r, t)=h=\mathrm{const}$ & 1 & $\theta$ & $t$ & $F(t)=F_{0} \cos ^{2} t$ \\
\hline
\end{tabular}

Заключение. Таким образом, представленные точные решения получены с помощью метода ИП, являющегося одним из подходов получения точных решений в рамках НСМ за счёт постулирования соответствия между координатами пространства-времени погружения и погружаемого пространства.

В рамках трёхкомпонентных сферически-симметричных моделей было получено два класса точных космологических решений. Одно из полученных точных решений для $h(r, t)=\mathrm{const}=1, \bar{g}_{33}=1$ и $x^{3}=t$ пространства- 
времени погружения представляют собой сферически-симметричные неоднородные метрики Толмена.

Кроме этого, впервые метод ИП был рассмотрен в рамках двухкомпонентных НСМ. На основании полученных данных были исследованы сферическисимметричные модели, для которых также получены два семейства точных решений: в общем случае при $x^{2}=r, x^{3}=t$ и $\epsilon=-1$ и в случае, когда $h(r, t)=$ const, при $x^{2}=t, x^{3}=\theta$ и $\epsilon=1$.

Работа выполнена при поддержке РФФИ (проект № 08-02-91307-ИНД_а) и ДСТ (проект RUS P/84-DST).

\section{БИБЛИОГРАФИЧЕСКИЙ СПИСОК}

1. Иванов Г. Г. Симметрии, законы сохранения и точные решения в нелинейной сигма-модели // ТМФ, 1983. Т. 57, № 1. С. 45-54; англ. пер.: Ivanov G. G. Symmetries, conservation laws, and exact solutions in nonlinear sigma models // Theoret. and Math. Phys., 1983. Vol. 57, no. 1. Pp. 981-987.

2. Иванов Г. Г. Полиномиальные законы сохранения и точные решения, связанные с изометрическими и гомотетическими симметриями, в нелинейной сигма-модели // TMФ, 1985. T. 62, № 1. С. 144-152; англ. пер.: Ivanov G. G. Polynomial conservation laws and exact solutions associated with isometric and homothetic symmetries in the nonlinear sigma model // Theoret. and Math. Phys., 1985. Vol.62, no.1. Pp. 95-101.

3. Chervon S. V. Chiral non-linear sigma model in general relativity and cosmology / In: Lecture Notes in Theoretical and Mathematical Physics. Kazan: Kazan State University Press, 2006. Pp. 108-172.

4. Chervon S., Dahia F., Romero C. Harmonic maps and isometric embeddings of the spacetime// Phys. Lett. A, 2004. Vol. 326, no. 3-4. Pp. 171-177, arXiv: gr-qc/0312022.

5. Chervon S., Romero C. The embedding of the space time in a target space of sigma model // Gen. Relativ. Gravitation, 2004. Vol. 36, no. 7. Pp. 1555-1561.

6. Червон C. В., Свистунова Ю. А. Генерирование точных решений в трехкомпонентной самогравитирующей кинетической нелинейной сигма-модели с использованием изометрических погружений// Изв. вуз. Поволжский регион. Физ.-мат. науки, 2008. № 2. C. 95-106. [Chervon S. V., Svistunova Yu. A. Generation of exact solutions in threecomponent self-gravitating kinetic non-linear sigma model with the use of isometric embeddings // Izv. Vuz. Povolzhskiy Region. Fiz.-Mat. Nauki., 2008. no. 2. Pp. 95-106].

7. Червон С.В. Плоско-симметричные решения в рамках $\mathrm{SO}(4)$-инвариантной самогравитирующей нелинейной сигма-модели // Изв. вуз. Физика, 1983. Т. 26, № 8. С. 8993. [Chervon S. V. Plane-symmetric solutions of $\mathrm{SO}(4)$-invariant non-linear self-gravitating sigma model // Izv. Vuz. Fizika, 1983. Vol. 26, no. 8. Pp. 89-93].

8. Червон C. В. Нелинейные поля в теории гравитации и космологии. Ульяновск: УлГУ Средне-Волжский НЦ, 1997. 191 с. [Chervon S. V. Non-linear fields in the theory of gravity and cosmology. Ul'yanovsk: UlGU - Sredne-Volzhskiy NC, 1997. 191 pp.]

9. Dahia F., Romero $C$. The embedding of the spacetime in five dimensions: an extension of Campbell-Magaard theorem // J. Math. Phys., 2002. Vol. 43, no. 11. Pp. 5804-5814.

10. Anderson E., Lidsey J. Embeddings in non-vacuum spacetimes // Class. Quant. Grav., 2001. Vol. 18, no. 22. Pp. 4831-4844.

11. Anderson E., Dahia F., Lidsey J., Romero C. Embeddings in spacetimes sourced by scalar fields // J. Math. Phys., 2003. Vol. 44, no. 11. Pp. 5108-5119.

12. Campbell J. A course of differential geometry. Oxford: Claredon Press, 1926. 261 pp.

13. Magaard L. Zur Einbettung Riemannscher Räume in Einstein-Räume und konformeuclidische Räume: PhD Thesis. Kiel, 1963.

14. Dahia F., Romero $C$. The embedding of the spacetime in five-dimensional spaces with arbitrary non-degenerate Ricci tensor // J. Math. Phys., 2002. Vol. 43, no. 6. Pp. 3097-3106. 
15. Tolman R. C. Relativity, thermodynamics, and cosmology. Oxford: Clarendon Press, 1934. 519 рр.; русск. пер.: Толмен Р. Относительность, термодинамика и космология. М.: Наука, 1974. 520 с.

16. Chervon S. V. Cosmological models of global universe evolution and decomposition of perturbations // Int. J. Mod. Phys. A, 2002. Vol. 17, no. 29. Pp. 4451-4456.

Поступила в редакцию 20/XII/2010;

в окончательном варианте - 26/IV/2011.

MSC: 83F05, 83E15

\section{SPHERICALLY-SYMMETRIC NON-LINEAR SIGMA MODEL: THE EXACT SOLUTIONS OBTAINED WITH ISOMETRICAL EMBEDDING METHOD}

\section{S. V. Chervon, Yu. A. Svistunova}

I. N. Ul'yanov's Ul'yanovsk State Pedagogical University,

4, pl. 100-letiya so dnya rozhdeniya V. I. Lenina, Ul'yanovsk, 432000, Russia.

E-mails: chervon.sergey@gmail.com, u.a.svistunova@gmail.com

The method to generate exact cosmological solutions in the frame of the sphericallysymmetric non-linear sigma model is offered in the present paper. This method is based on the isometrical embeddings of the target space (non-linear sigma-model fields chiral space) into space-time. Also the method of application to two- and three-component chiral spaces embedded into space-time was considered. The exact cosmological solutions were obtained in the frame of the several special cases of the two-and three-component spherical-symmetric non-linear sigma-model. The obtained cosmological solutions were also investigated.

Key words: non-linear sigma model, isometrical embedding method, exact cosmological solutions.

Original article submitted 20/XII/2010; revision submitted $26 / \mathrm{IV} / 2011$.

Sergey V. Chervon (Dr. Sci (Phys. \& Math.)), Professor, Dept. of Physics. Yulia A. Svistunova, Researcher, Dept. of Physics. 